\title{
A case of eribulin-induced regression of liposarcoma of the left funiculus in a heavily pretreated patient
}

\author{
Salvatora Tindara Miano*,1, Edoardo Francini ${ }^{2,3}$, Roberto Petrioli ${ }^{1}$ \& Guido Francini \\ ${ }^{1}$ Oncology Unit, Policlinico Santa Maria Alle Scotte, Università degli Studi di Siena, Siena, Italy \\ ${ }^{2}$ Medical Oncology Unit, Misericordia Hospital, Grosseto, Italy \\ ${ }^{3}$ Department of Medical-Surgical Sciences \& Translational Medicine, University of Rome "La Sapienza", Rome, Italy \\ *Author for correspondence: Tel.: +3905 7758 5418; Fax +3905 7758 6231; doramiano@hotmail.com
}

We report the case of a heavily pretreated male subject affected by left funiculus liposarcoma and successfully treated with eribulin mesylate.

After three surgical interventions, radiotherapy on the lesion of the penile bulb for satellite nodules and an epirubicin + ifosfamide chemotherapy treatment for six cycles, eribulin was administered at the dose of $1.1 \mathrm{mg} / \mathrm{m}^{2}$ on days 1 and 8 , every 3 weeks for a total of nine cycles. A significant reduction of the lesions was achieved after four cycles of therapy, with a good profile of tolerability.

First draft submitted: 24 September 2019; Accepted for publication: 8 January 2020; Published online: 24 January 2020

Keywords: eribulin mesylate $\bullet$ left funiculus $\bullet$ liposarcoma $\bullet$ tolerability $\bullet$ epirubicin $\bullet$ ifosfamide $\bullet$ radiotherapy

The management of soft tissue sarcomas (STS) is challenging, mainly due to their heterogeneity. The standard first-line chemotherapy for advanced STS is based on doxorubicin, with or without ifosfamide; however, a number of different drugs are emerging as active therapeutic agents beyond the doxorubicin first-line therapy. Eribulin mesylate is a structurally modified analog of halichondrin B, able to act as a potent microtubule-destabilizing anticancer agent. It has been recently approved for the treatment of advanced liposarcoma (LPS) following an anthracycline-based regimen, demonstrating an overall survival (OS) advantage in LPS and leiomyosarcoma in a randomized Phase III clinical trial [1]. Here we report the case of a male patient affected by LPS of the left funiculus and treated with eribulin mesylate after a series of surgical and drug treatments (Figure 1). This therapeutic approach and the resulting data in terms of effectiveness and tolerability represents, to the best of our knowledge, a novelty in the setting of LPS affecting the left funiculus in a heavily pretreated patient.

\section{Case presentation}

We describe the case of a Caucasian male patient born in 1942, affected by LPS of the left funiculus, that is in follow-up.

In June 2012 the patient was admitted to General Surgery of the P.O. Amiata Senese (Siena, Italy) with a diagnosis of external oblique inguinal hernia and left solid parafunicular mass with a diameter of $3 \mathrm{~cm}$. The patient was submitted to left inguinal hernioplasty with prosthesis and to enucleation of the parafunicular mass. The overall findings from the histopathological examination suggested an inflammatory myofibroblastic tumor (proliferation of fused, ganglion-like cellular elements, calponin ${ }^{+}, \mathrm{actin}^{-}, \mathrm{alk}^{+}, \mathrm{mdm} 2^{+}, \mathrm{cd} 8^{+}$, rare mitotic events, mild atypia, $10 \% \mathrm{Ki}^{+} 7^{+}$cells, moderate inflammatory infiltrate).

A testicular ultrasound performed in March 2013 revealed a solid hypoechoic oval mass with irregular margins proximal to the left inguinal canal, on the external side, $2 \times 1.6 \mathrm{~cm}$ in size, apparently in continuity with the structures of the spermatic cord. A bilateral hydrocele of modest size was observed as well.

An abdomen CT scan performed in April 2013 revealed a nonhomogeneous mass at the left spermatic cord, partly attributable to the outcomes of the surgical intervention and partly to a relapse of the disease, in particular at level of the pubic symphysis and along the scrotal sac. No abdominal or inguinal lymphadenopathy was detected.

Future 8 Medicine 


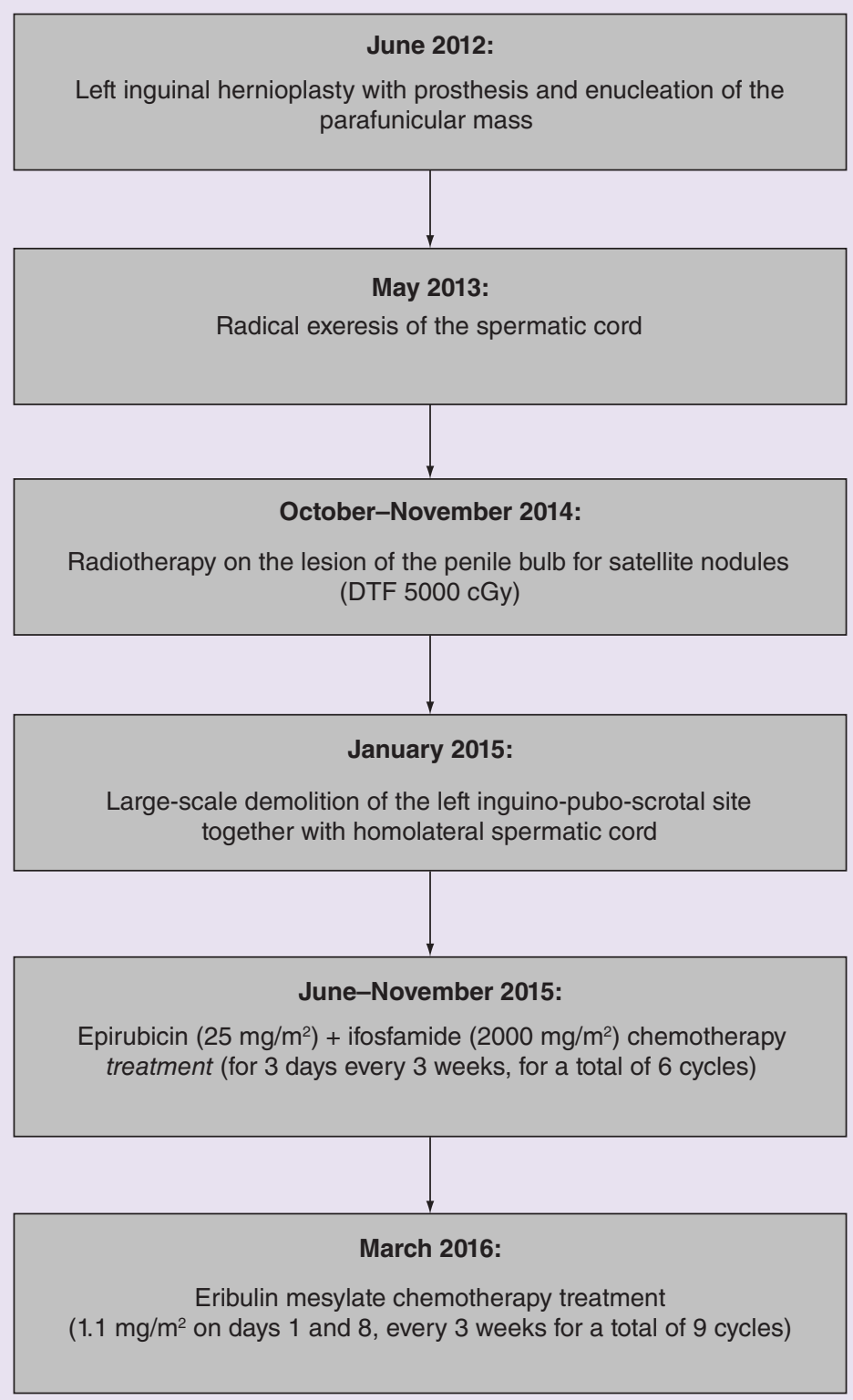

Figure 1. Flowchart of dose and timeline of treatments in a male patient affected by liposarcoma of the left funiculus.

In May 2013, the patient was admitted to the General Surgery of the P.O. Amiata Senese for an intervention of radical exeresis of the spermatic cord, affected by tenacious fibrosis and suspected for myofibroblastic recurrence, and of the left testicle. The histopathological examination revealed areas of well-differentiated LPS of sclerosing inflammatory type and atypical lipoma of the soft tissues of the funiculus, didymus, testis network and uninjured epididymal site. The final diagnosis was of dedifferentiated LPS.

An abdomen CT scan performed in November 2013 revealed an increase of some preaortic, subrenal lymph nodes, with the greatest one about $9.45 \mathrm{~mm}$ (ex seven $\mathrm{mm}$ ) in size, together with a modest increase in the amount of tissue after the surgical intervention. No other pathological findings compatible with the disease were found.

An abdomen CT scan performed in August 2014 revealed conspicuous local disease as outcome of the surgery for left spermatic cord neoplasia due to size increase of the previously described solid tissue; the lateroaortic adenopathies described above were unchanged and probably of nonevolutionary significance. 


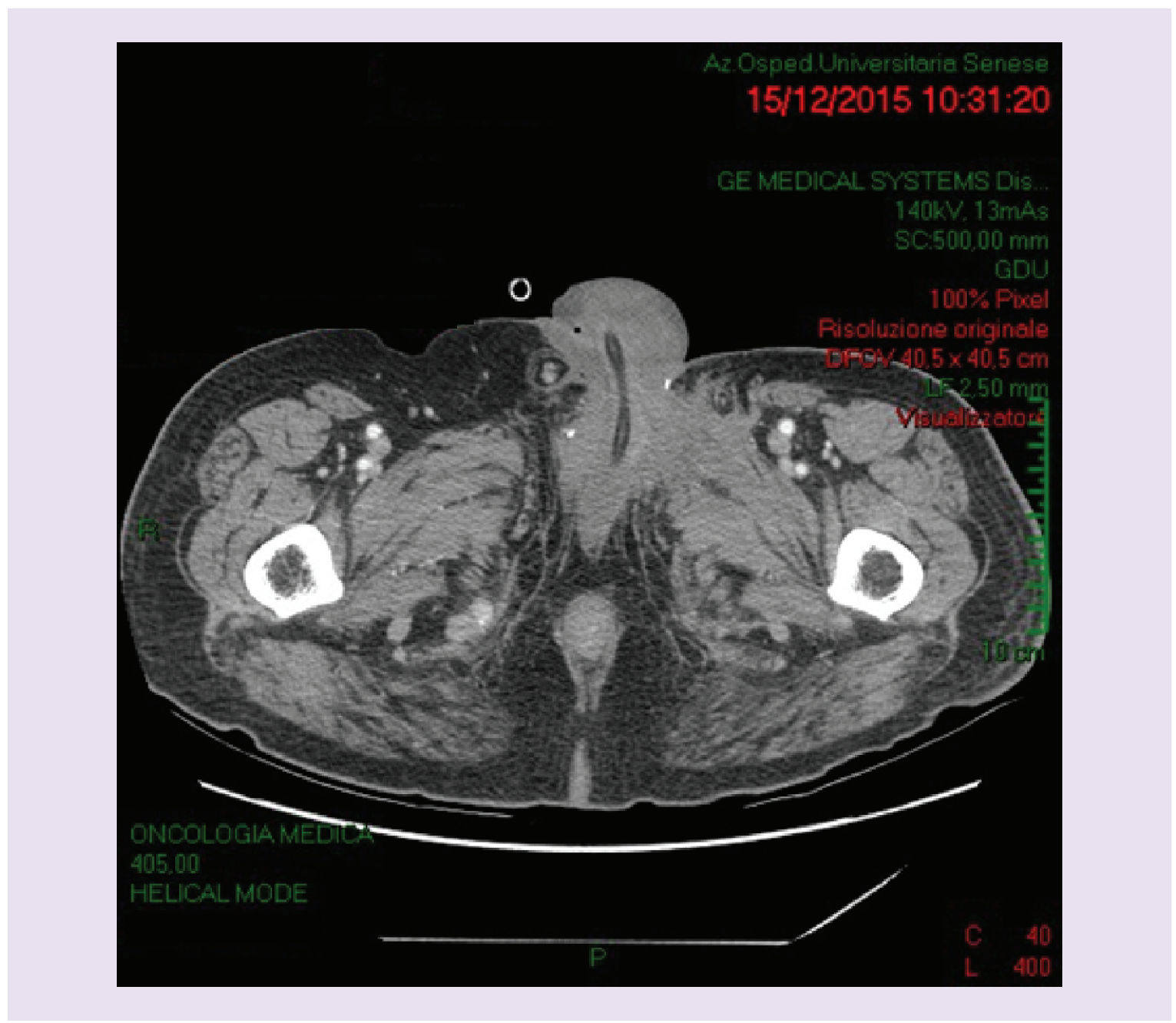

Figure 2. Abdomen CT scan performed in December 2015 showing a pathological nodule of maximum $25 \mathrm{~mm}$ in size along the left gonadal vessels, about $6 \mathrm{~cm}$ from the outlet in the ipsilateral renal vein.

From October 2014 to November 2014 the patient underwent radiotherapy on the lesion of the penile bulb for satellite nodules (DTF $5000 \mathrm{cGy}$ ).

A thorax and abdomen CT scan performed in December 2014 revealed a minimum increase (overall $<20 \%$ ) of the known hypervascular lesions of the subcutaneous adipose tissue in the region between the left inguinal canal and the root of the penis, and above all of the region anterior to the pubic symphysis, with signs of infiltration of the left pectineus muscle. The small lymph nodes remained unchanged bilaterally in the groin. Pathological lymphadenopathy was not evident in secondary abdominal or thoracic lesions.

In January 2015 the patient was admitted to the Surgery Department of another P.O. for a large-scale demolition of the left inguino-pubo-scrotal site together with homolateral spermatic cord, splint of pubic symphysis, ileopubic branch and root of cavernous bodies of the penis and for reconstruction by local flaps.

In June 2015 the patient started a chemotherapy treatment with epirubicin, administered at the dose of $25 \mathrm{mg} / \mathrm{m}^{2}$, and ifosfamide, at the dose of $2000 \mathrm{mg} / \mathrm{m}^{2}$, for three days every 3 weeks, for six cycles (the last one in November 2015).

A chest and abdomen CT scan performed in December 2015 (Figure 2) showed a pathological nodule of maximum $25 \mathrm{~mm}$ in size along the left gonadal vessels, about $6 \mathrm{~cm}$ from the outlet in the ipsilateral renal vein. No other pathological findings compatible with disease locations were detectable in the anatomical districts examined.

An abdomen CT scan performed in February 2016 showed no signs of locoregional recurrence of the disease; however, there was a minimal hypervascularization in the area of the penis and the presence of scar tissue between 


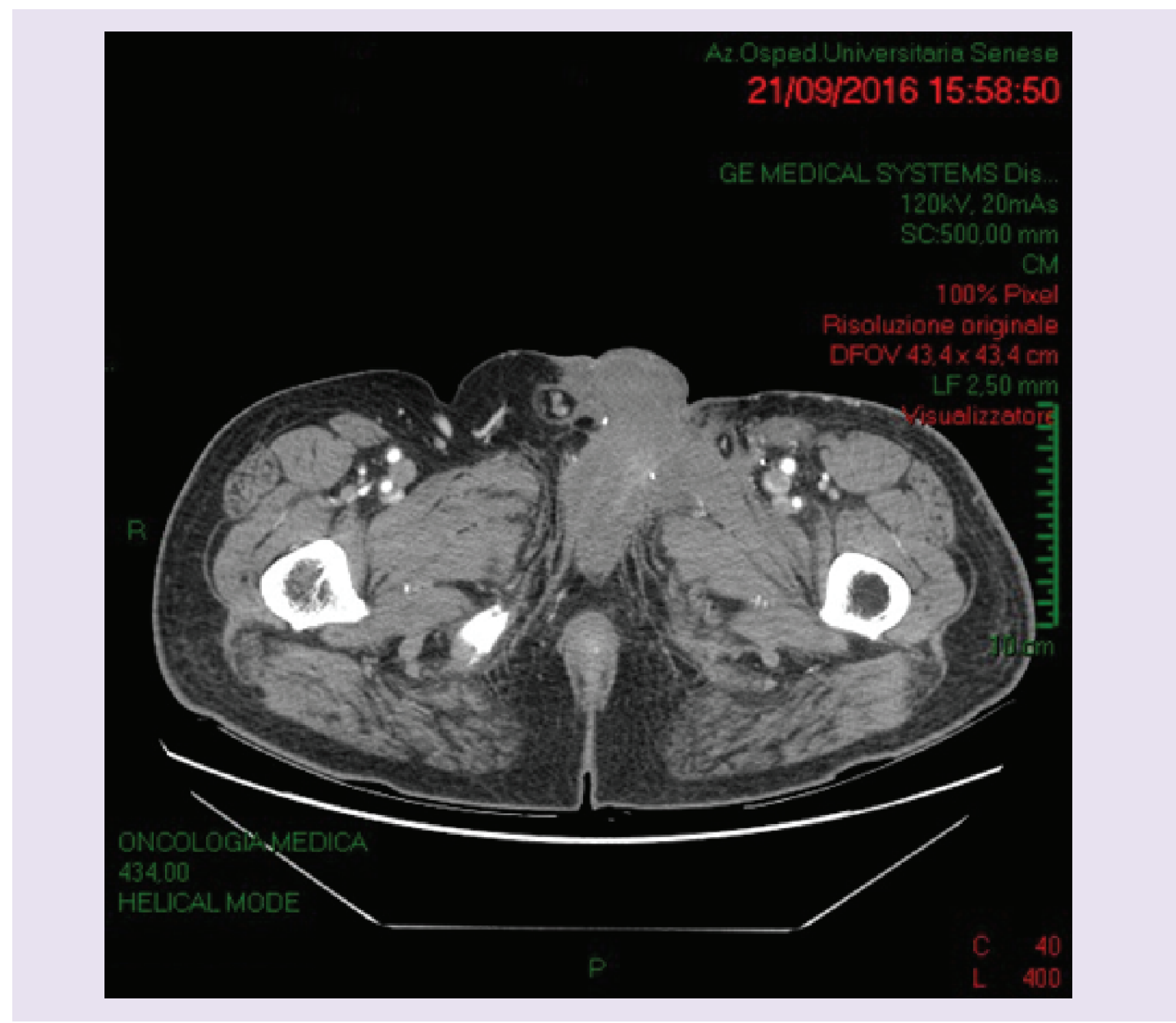

Figure 3. Abdomen CT scan performed in September 2016 after eribulin treatment and revealing the outcomes of the recent surgery at level of the inguinal region, with a reduction of the homolateral hernia (residual herniation of the bladder) and evidence of widespread soft tissue irregularity with hypervascularized areas.

the root of the penis and the left inguinal canal, requiring a close follow-up. There were no morphological and size changes of the nodule previously observed along the left gonadal vessels. No secondary pulmonary or hepatic injuries were detected.

In March 2016 the patient started a chemotherapy regimen with eribulin mesylate, administered at the dose of $1.1 \mathrm{mg} / \mathrm{m}^{2}$ on days 1 and 8 , every 3 weeks for a total of nine cycles. A significant reduction of the lesions was achieved after four cycles of therapy, with a good profile of tolerability. A CT scan performed in September 2016 (Figure 3) revealed the outcomes of the recent surgery at level of the inguinal region, with a reduction of the homolateral hernia (residual herniation of the bladder) and evidence of widespread soft tissue irregularity with hypervascularized areas. However, due to the short interval of time elapsed, a reliable differential diagnosis between post-surgical outcomes (more probable) and disease relapse could not be given.

\section{Discussion}

Sarcomas represent about $1 \%$ of all adult malignancies; they are a heterogeneous group of solid tumors derived from mesenchymal progenitor cells [2]. Approximately $80 \%$ of sarcomas originate from viscera or soft tissue, while $20 \%$ of cases originate from the bone. More than 50 different histological subtypes of STSs have been currently classified [3], with an incidence rate of about three to five cases per 100,000/year [2,4]. LPSs are one of the most common STS subtypes in adult subjects and are classified on the basis of their molecular, clinical 
and morphological/histological features. Dedifferentiated, well-differentiated, pleomorphic and myxoid/round cell variants are included among LPS subtypes [5]. Current therapeutic options for patients affected by all types of advanced STSs are limited, with an increasing relevance of histology-based management of the disease. Cytotoxic chemotherapy remains the first-line treatment for the majority of patients affected by advanced or metastatic STS, other than by gastrointestinal (GI) stromal tumors or by dermatofibrosarcoma protuberans, with variations in the clinical practice between doxorubicin as monotherapy or in combination with dacarbazine or ifosfamide. Eribulin mesylate has been originally isolated from the marine sponge Halichondria okadai; it is an analog of halichondrin B with structural modifications. Eribulin mesylate is a nontaxane inhibitor of microtubule dynamics with a peculiar mechanism of action involving the binding to specific sites on the microtubule growing plus $(+)$ ends $[6,7]$. Eribulin mesylate was able to induce vascular remodeling and tumor cell differentiation in preclinical models of breast carcinoma and sarcomas, suppressing cell migration and invasion, and reversing the epithelial-to-mesenchymal transition (in carcinomas) [8-10]. Eribulin mesylate has been approved for the therapy of advanced or metastatic breast cancer in patients that received at least two (USA) or one (EU) prior chemotherapy regimen(s) [11,12]. In a randomized, Phase III prospective, international study enrolling patients affected by unresectable, advanced or metastatic LMS or LPS after the failure of a previous chemotherapy regimen, eribulin mesylate induced a significant improvement of OS versus dacarbazine (13.5 vs 11.5 months, respectively; HR: 0.77; 95\% CI: 0.62$0.95 ; \mathrm{p}=0.0169)$ and exhibited a manageable toxicity profile, overlapping with the profiles observed in patients affected by breast cancer [13]. The benefits in terms of OS obtained with eribulin mesylate vs. dacarbazine were greater in patients affected by LPS than in those affected by LMS. Of interest, a recent interim analysis of a post-marketing surveillance study revealed that eribulin mesylate is generally well tolerated and shows antitumor activity also against rare subtypes of STS, that currently have few effective treatment options [14]. Finally, novel data obtained in a patient-derived orthotopic xenograft murine model of osteosarcoma revealed the effectiveness of eribulin mesylate in arresting the tumor growth [15], thus potentially increasing the therapeutic targets of this drug.

The case we reported here is affected by the lack of a long term follow-up on the patient. Nonetheless, we extended the current knowledge showing the good tolerability and the efficacy of an eribulin-based therapeutic regimen in the case of a male patient affected by LPS of the left funiculus and heavily pretreated.

Summary points

- The management of soft tissue sarcomas (STSs) is challenging, mainly due to their heterogeneity.

- Current therapeutic options for patients affected by advanced STSs are limited, with an increasing relevance of histology-based management of the disease.

- We described the case of a Caucasian male patient affected by LPS of the left funiculus, that is currently in follow-up.

- The patient has been submitted to a total of three surgical interventions, from 2012 to 2015.

- In 2014 the patient has been treated for one month with radiotherapy on the lesion of the penile bulb for satellite nodules.

- In 2015 the patient started an epirubicin + ifosfamide chemotherapy treatment for six cycles.

- In 2016 the patient started a chemotherapy regimen with eribulin mesylate, for a total of nine cycles.

- A significant reduction of the lesions was achieved already after four cycles of eribulin mesylate therapy, with a good profile of tolerability.

Author contributions

All authors were involved in the writing and revision of this manuscript and have provided final approval to submit.

Financial \& competing interests disclosure

The authors have no relevant affiliations or financial involvements with any organization or entity with a financial interest in or a financial conflict with the subject matter or materials discussed in the manuscript. This includes employment, consultancies, honoraria, stock ownership or options, expert testimony, grants or patents received or pending, or royalties.

Medical writing support was provided by A Forte on behalf of Content Ed Net, and funded by Eisai. 


\section{Ethical conduct of research}

The authors state that they have followed the principles outlined in the Declaration of Helsinki for all human or animal experimental investigations. In addition, for investigations involving human subjects, informed consent has been obtained from the participants involved

\section{References}

Papers of special note have been highlighted as: $\bullet \bullet$ of considerable interest

1. Setola E, Noujaim J, Benson C, Chawla S, Palmerini E, Jones RL. Eribulin in advanced liposarcoma and leiomyosarcoma. Expert Rev. Anticancer. Ther. 17(8), 717-723 (2017).

-. A comprehensive review of the efficacy and safety of eribulin in soft tissue sarcomas (STS) is presented, with a focus on the different clinical outcomes among histological subtypes.

2. National Comprehensive Cancer Network. National Comprehensive Cancer Network Clinical Practice Guidelines in Oncology: NCCN guidelines: Soft tissue sarcoma (2019). www.nccn.org/professionals/physician_gls/default.aspx

3. Fletcher CDM, Bridge JA, Hogendoorn P, Mertens F. WHO Classification of Tumours of Soft Tissue and Bone (ed 4). IARC Press, Lyon, France (2013).

4. ESMO/European Sarcoma Network Working Group. Soft tissue and visceral sarcomas: ESMO Clinical Practice Guidelines for diagnosis, treatment and follow-up. Ann. Oncol. 25(Suppl. 3), iii102-12 (2014).

5. Ducimetière F, Lurkin A, Ranchère-Vince $\mathrm{D}$ et al. Incidence of sarcoma histotypes and molecular subtypes in a prospective epidemiological study with central pathology review and molecular testing. PLoS One 6(8), e20294 (2011).

6. Dybdal-Hargreaves NF, Risinger AL, Mooberry SL. Eribulin mesylate: mechanism of action of a unique microtubule-targeting agent. Clin. Cancer Res. 21(11), 2445-2452 (2015).

7. Smith JA, Wilson L, Azarenko $\mathrm{O}$ et al. Eribulin binds at microtubule ends to a single site on tubulin to suppress dynamic instability. Biochemistry 49(6), 1331-1337 (2010).

8. Funahashi Y, Okamoto K, Adachi Y et al. Eribulin mesylate reduces tumor microenvironment abnormality by vascular remodeling in preclinical human breast cancer models. Cancer Sci. 105(10), 1334-1342 (2014).

9. Yoshida T, Ozawa Y, Kimura T et al. Eribulin mesilate suppresses experimental metastasis of breast cancer cells by reversing phenotype from epithelial-mesenchymal transition (EMT) to mesenchymal-epithelial transition (MET) states. Br. J. Cancer 110(6), 1497-1505 (2014).

10. Kawano S, Asano M, Adachi Y, Matsui J. Antimitotic and non-mitotic effects of eribulin mesilate in soft tissue sarcoma. Anticancer Res. 36(4), 1553-1561 (2016).

11. Eisai: Halaven (eribulin mesylate) injection [prescribing information]. Eisai, Woodcliff Lake, NJ (2016).

12. Eisai: Halaven $0.44 \mathrm{mg} / \mathrm{ml}$ solution for injection [summary of product characteristics]. Eisai Europe Limited, Hertfordshire, UK.

13. Schöffski P, Chawla S, Maki RG et al. Eribulin versus dacarbazine in previously treated patients with advanced liposarcoma or leiomyosarcoma: a randomised, open-label, multicentre, Phase 3 trial. Lancet 387(10028), 1629-1637 (2016).

-. The summary of a Phase III study, aiming to compare the overall survival in patients with advanced or metastatic soft-tissue sarcoma who received eribulin versus patients who received dacarbazine.

14. Kobayashi E, Naito $\mathrm{Y}$, Asano $\mathrm{N}$ et al. Interim results of a real-world observational study of eribulin in soft tissue sarcoma including rare subtypes. Jpn. J. Clin. Oncol. 49(10), 938-946 (2019).

15. Kiyuna T, Tome Y, Miyake $\mathrm{K}$ et al. Eribulin suppressed cisplatinum- and doxorubicin-resistant recurrent lung metastatic osteosarcoma in a patient-derived orthotopic xenograft mouse model. Anticancer Res. 39(9), 4775-4779 (2019). 